\title{
Modulation of surface wettability by vibration assisted milling
}

\author{
Lu Zhenga ${ }^{a}$, Wanqun Chen ${ }^{a}$,, Michele Pozzi ${ }^{a}$, Xiangyu Teng ${ }^{a}$, Dehong Huo ${ }^{a *}$
}

\begin{abstract}
a. Mechanical Engineering, School of Engineering, Newcastle University, Newcastle upon Tyne, NE1 7RU, UK
b. Centre for Precision Engineering, Harbin Institute of Technology, Harbin 150001, P. R. China
\end{abstract}

\begin{abstract}
Surface textures have significant influence on surface wettability. In this paper, a range of fish scales surface textures are produced by using vibration assisted micro milling. The generation principle of the surface textures and their influence on surface wettability are mathematically studied. A new contact model, which considers both liquid infiltration effects and air trapped in the microstructure, is proposed for predicting the wettability of the fish scales surface texture. Machining and hydrophobicity experiments are conducted to validate the model and results indicate that the solid surface hydrophilicity is improved by the fish scales surface textures. Moreover, under the experimental conditions used in this research, the contact angle can be controlled from $30^{\circ}$ to $57^{\circ}$ by adjusting the parameters that determine the fish scales. The paper concludes that vibration assisted micro milling is an effective method to modify and control surface wettability.
\end{abstract}

Keywords: vibration assisted milling, micro milling, surface texture, wettability, contact mode

\section{Introduction}

Wettability is one of the most important characteristics inherent to a surface, which has a significant effect on the flow of a liquid over a solid substrate [1]. It is the result of the interfacial tensions interaction of three phases (vapour, solid surface and liquid) and can be expressed by the surface contact angle [2]. Benefits such as superior lubrication and wear resistance can be obtained by modifying a solid surface wettability to an appropriate value, which results in wide applications such as in the bio-medical field and oil industry [3-6]. Surface wettability can be affected by many factors, such as material types, surface roughness and surface microstructure. Accordingly, research on creating particularly hydrophilic surfaces and the relevant theories, such as contact models and the influence of surface free energy variation on contact angle have been carried out in the past decades. Li et al. [7] found that

\footnotetext{
* Corresponding author: D. Huo, Tel.: +44 (0)191 2086230

E-mail address: dehong.huo@newcastle.ac.uk
} 
solid surface hydrophilicities with distinct surface textures can be quite different even if they have similar surface roughness. To explain this phenomenon, an analytical method was proposed to calculate the contact angle based on a typical pillar surface microstructure by considering the surface energy variation in the Wenzel and Cassie's contact model. A different theory is proposed by Shi et al. [8]: they fabricated a parallel-channels surface microstructure by using high speed micro milling machine and built a relationship between the droplets' Gibbs free energy on the surface and the contact angle. To improve the hydrophilicity of a polymer membrane and extend its life, Yang et al. [9] fabricated a mineral coating to cover it. Results showed that both wettability and structural stability of the polymer membrane were improved. However, this method cannot produce a controllable wettability of the surface. Fuat et al. [10] solved this problem by developing a multilayers coating. Through changing the terminal layer, the contact angle varies between $37^{\circ}$ and $45^{\circ}$. However, its application is limited by the complexity of the process, its high cost and the coating's susceptibility to ageing. Producing surface textures is another way to change surface drop impact characteristics, and many techniques such as high speed micro machining, laser cutting and etching have been applied in the manufacturing process. A pillars-array microstructure fabricated with a CNC machine is proposed by Lee et al. [11]. It is found that the pillars-array micro-structure can effectively improve the material hydrophobic properties and the surface wetting state changes (from Cassie state to Wenzel state) as pillars' spacing increases. Laser cutting is another efficient way to manufacture unique surface patterns with different surface wettability. However, special equipment is required for the process [12,13]. Andrew et al. [14] developed a parallel-grooves surface structure by using etching. According to the contact angle measurement results, the etched surface hydrophobicity is increased and can be controlled within a certain range; furthermore, an anisotropic wettability is obtained. the surface textures obtained by using nontraditional machining techniques also suffer from problems such as hard to control production process and hence inaccuracy in reproduction [15]. Although non-traditional machining techniques permit tailoring wettability via surface textures, they involve complex production processes and are difficult to reproduce accurately [15].

As a new machining method, vibration assisted micro milling (VAMM), featuring a periodic separation between the workpiece and the tool, offers numerous advantages such as lower cutting force, longer tool life, better surface quality, and higher machining accuracy [1621]. VAMM has found applications in processing hard and brittle materials such as silicon, 
titanium alloy and ceramics [22]. Moreover, with an appropriate selection of parameters, unique surface textures can be created by VAMM.

In this study, a range of fish scales surface textures are produced by vibration assisted micro milling on aluminium alloy. The surface texture formation mechanism is modelled by studying the kinematics locus of the cutting end of the two-flute milling tool. A series of machining experiments was carried out to generate surface textures with different characteristic dimensions. A new contact model is proposed to predict the resulting wettability. Sessile drop experiments are conducted to assess the accuracy of the new contact model.

\section{Kinematic analysis of surface topography generation in vibration assisted micro}

\section{milling}

\subsection{Tool tip trajectories analysis}

The principle of surface structure modification during vibration assisted micro milling is investigated in this paper. As it is performed by adding high-frequency vibration to the precision micro end milling process, the exact trajectories of the cutting edges of a two-flute milling tool in the conventional milling process are firstly studied. Fig.1 illustrates such trajectories, represented by solid and dashed curves. The mathematical equations of the tool tips motion are:

$$
\left\{\begin{array}{c}
X_{i}=V_{f} t+R \sin \left(\frac{n \pi t}{30}+i \pi\right) \\
Y_{i}=R \cos \left(\frac{n \pi t}{30}+i \pi\right)
\end{array}\right.
$$

Where $X_{i}$ and $Y_{i}$ represent the coordinates of the tool tip positions, $i$ indexes the two cutting edges ( 0 and 1$), V_{f}(\mathrm{~mm} / \mathrm{s})$ is the cutting speed, $n(\mathrm{rev} / \mathrm{min})$ is the spindle speed, $R(\mathrm{~mm})$ is the tool radius and $t(\mathrm{~s})$ is the cutting time. 


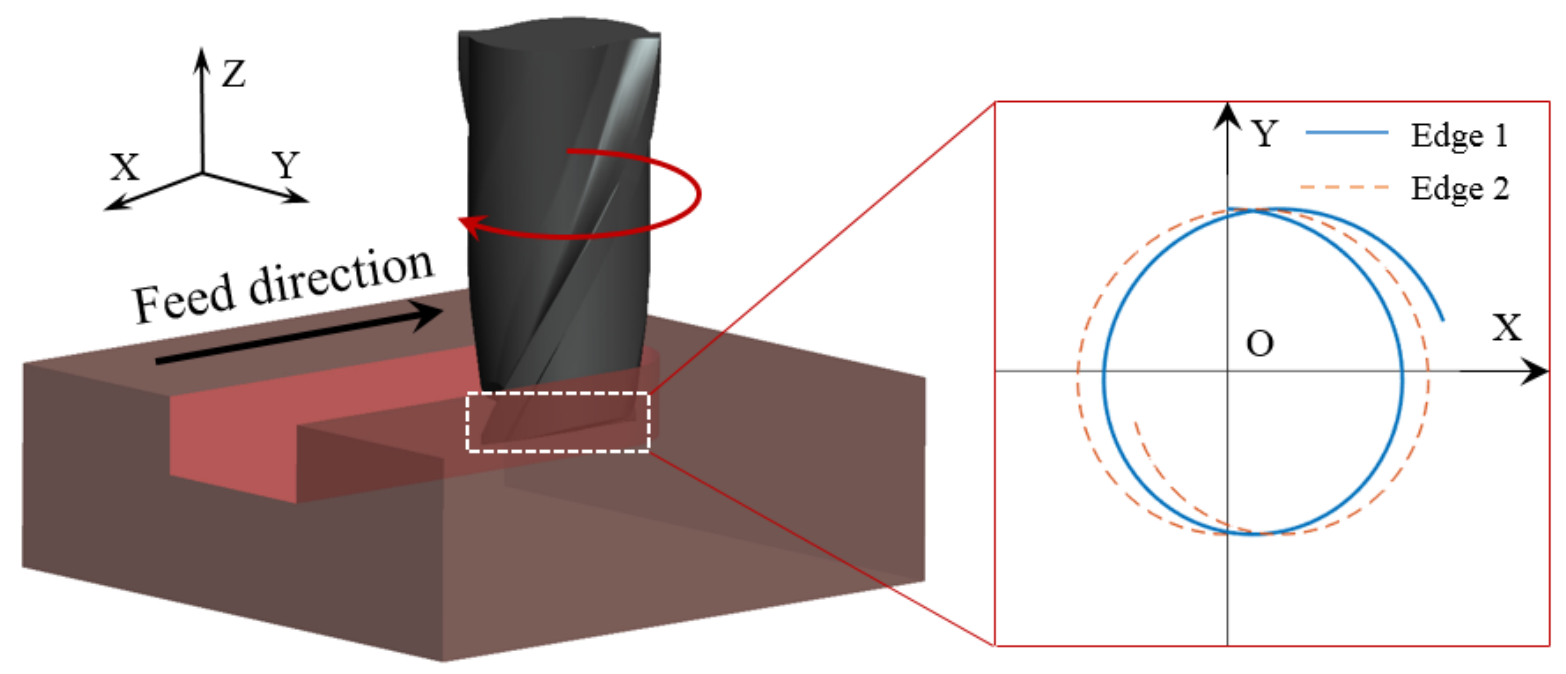

Figure 1 Conventional end milling tool tip trajectories

When an $X$ axis direction (feed direction) sinusoidal signal vibration is applied to the process, the new tool tips trajectories can be expressed as:

$$
\begin{gathered}
X_{i}=V_{f} t+R \sin \left(\frac{n \pi t}{30}+i \pi\right)+A_{x} \sin \left(2 \pi f_{x} t+\varphi_{x}\right) \\
Y_{i}=R \cos \left(\frac{n \pi t}{30}+i \pi\right)
\end{gathered}
$$

Where $f_{x}(\mathrm{~Hz})$ and $A_{x}(\mu \mathrm{m})$ are the vibration frequency and amplitude, respectively, and $\varphi_{x}$ is a phase angle.

According to Eq. (2), two unique adjacent tool paths can be generated, as shown in Fig. 2. With proper phase differences, a closed area (fish scales) can be produced, with the crests or troughs of the one tool tip corresponding to the troughs or crests respectively of the following tool tip. To describe this situation, the ratio between vibration frequency and spindle rotational frequency is defined as the frequency ratio $(\delta)$ and the condition to achieve this regular pattern for a two-flute cutter is:

$$
\text { Frequency ratio }(\delta)=60 f_{\mathrm{x}} / \mathrm{n}=\text { odd numbers }
$$




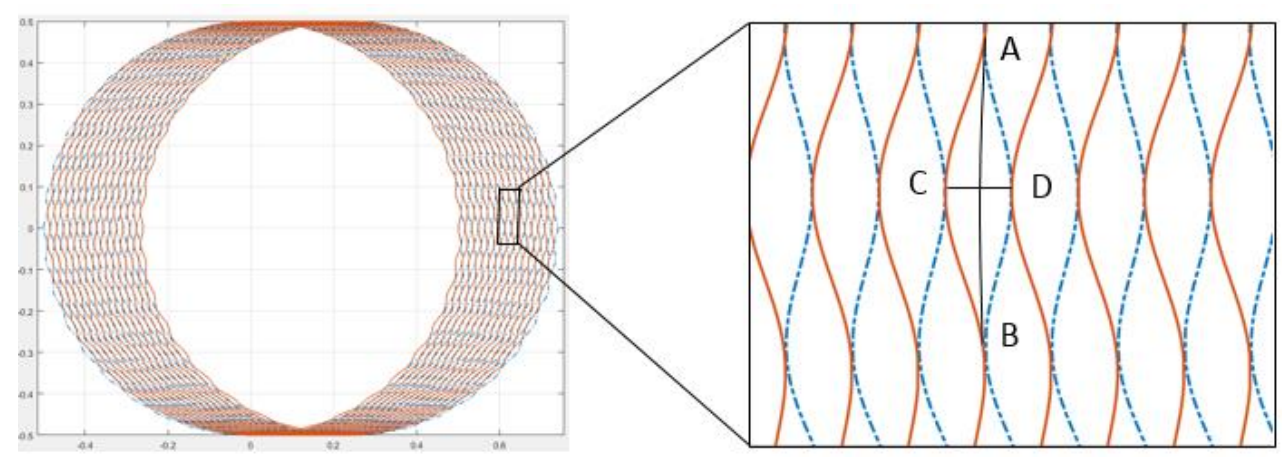

Figure 2 Tool tip trajectories for vibration assisted milling

By considering the relationship between vibration amplitude and feed rate $f_{z}$ ( $\mu \mathrm{m} /$ tooth), three types of fish scales surface textures can be fabricated, each with specific characteristic dimensions ( $A B, C D$ in Fig. 3).

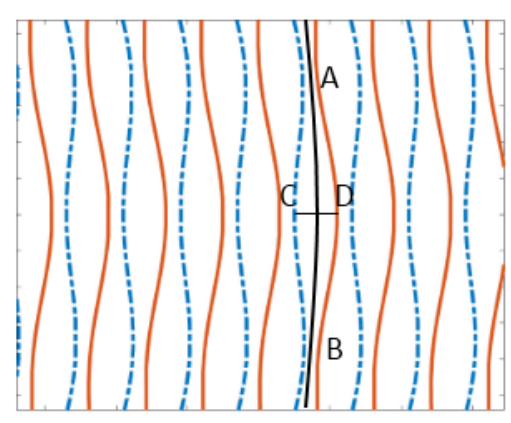

(a) $\quad A<f_{z} / 2$

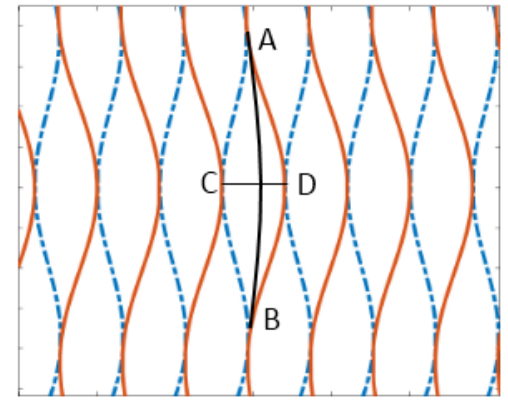

(b) $\quad A=f_{z} / 2$

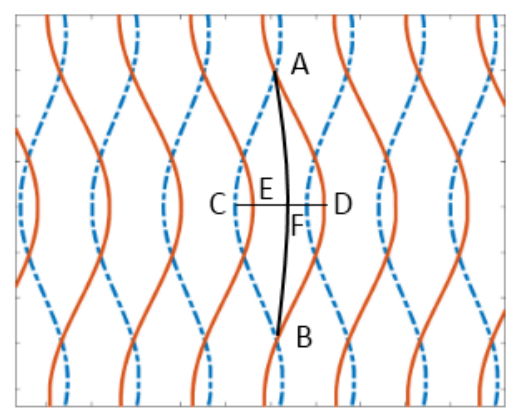

(c) $f_{z} / 2<A \leq f_{z}$

Figure 3 Different topography on fish scales surface structure

When the vibration amplitude is less than half of the feed rate, there is no intersection and a gap can be found between one and the following tool tips trajectories, see Fig.3(a). The area of the fish scales decreases as the vibration amplitude is reduced. The characteristic dimensions can be expressed as:

$$
\left\{\begin{array}{c}
\widehat{A B}=\pi R n / 60 f_{x} \\
C D=2 A_{x}
\end{array}\right.
$$

Fig.3(b) illustrates the second type of fish scales surface structure on the condition of $A=$ $f_{z} / 2$. Intersection points can be found in the tool tips trajectories and the characteristic dimensions can be expressed again using Eq. (4). 
Fig.3(c) shows the third type of fish scales surface structure, obtained when $\frac{f_{Z}}{2}<A<f_{z}$. The crest, or trough, of the former tool tooth overlaps with the trough, or crest, of the following tool, so the characteristic dimensions can be expressed as:

$$
\left\{\begin{array}{c}
\widehat{A B}=\pi R n / 60 f_{x} \\
C E=F D=A_{x}-f_{z} / 2 \\
E F=2 f_{z} \\
C D=2 A_{x}+f_{z}
\end{array}\right.
$$

It can be noted that the length $\widehat{\mathrm{AB}}$ of the surface texture is in proportion to the tool radius and the spindle speed, and in inverse proportion to the vibration frequency. The width $C D$ of the surface texture increases with the increase of the feed per tooth and the amplitude of the vibration.

\subsection{Depth prediction in fish scales surface textures}

The analysis of the depth profile of fish scales surface structures requires understanding of the surface generation mechanism. In the conventional micro-milling process, the cutter path has a great effect on the formation of tool marks and affects the surface topography. Depending on cutter tooth profile and cutting parameters, the feed marks assume one of two configurations, as shown in Fig.4.

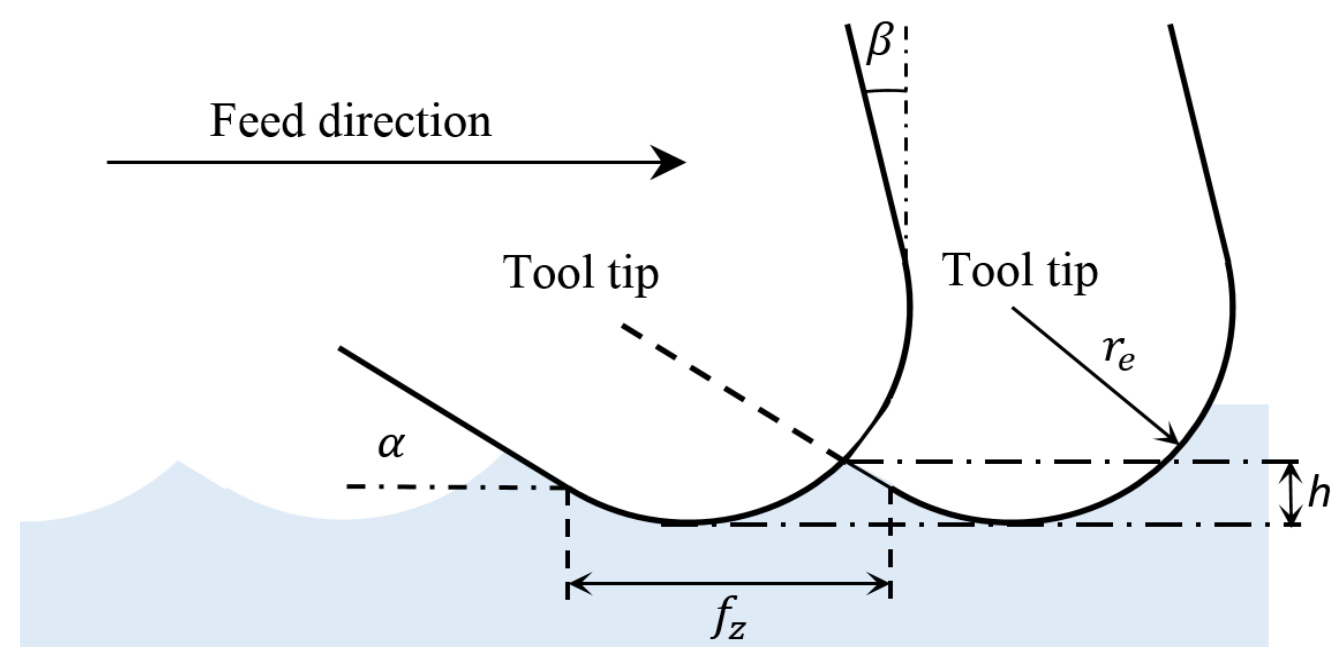

(a) 


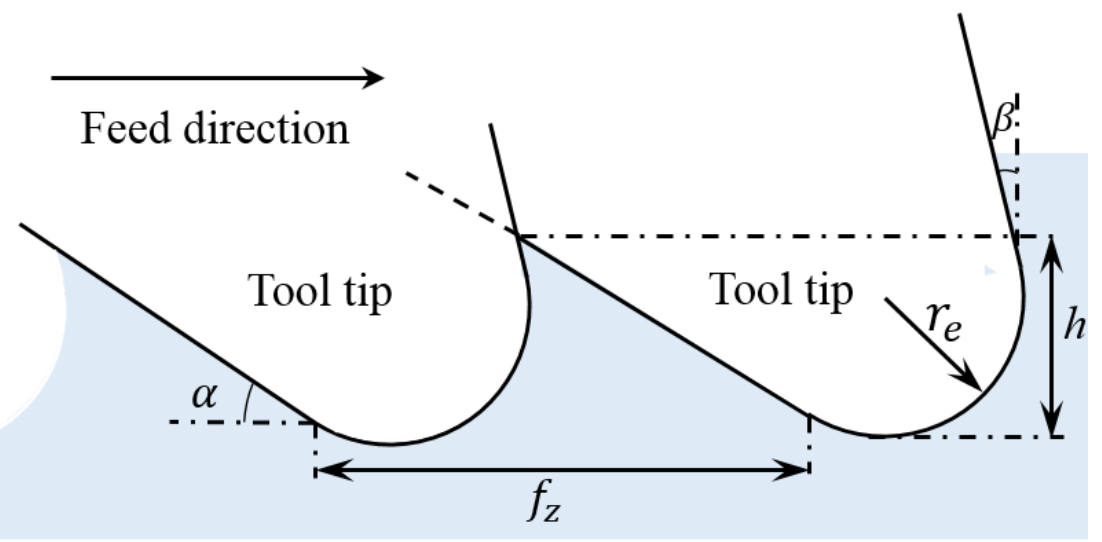

(b)

Figure 4 tool mark generation process

Fig. 4(a) shows the successive positions of the tool tip when $f_{z} \leq r_{e} \cos \beta+\frac{r_{e} \sin \beta}{\tan \alpha}+$ $\frac{r_{e} \cos ^{2} \alpha}{\sin \alpha}+r_{e} \sin \alpha:$ the current tool edge and the flank face of the following tool tooth intersect in a point that determines the height of the structure. The feed mark height $h$ can be expressed as:

$$
h=\frac{4 r_{e}+r_{e}^{2}-\left(\left(f_{z}-\frac{r_{e} \cos ^{2} \alpha}{\sin \alpha}-r_{e} \sin \alpha\right)^{2}-\sqrt{-4\left(f_{z}-\frac{r_{e} \cos ^{2} \alpha}{\sin \alpha}-r_{e} \sin \alpha\right)^{2}-2 r_{e}\left(f_{z}-\frac{r_{e} \cos ^{2} \alpha}{\sin \alpha}-r_{e} \sin \alpha\right)+8 r_{e}^{2}}\right)}{4}
$$

Where $r_{e}$ is the cutting edge radius, $\alpha$ and $\beta$ are the tool edge clearance angle and tool edge rake angle, respectively.

Fig. 4(b) shows the condition when $f_{z}>r_{e} \cos \beta+\frac{r_{e} \sin \beta}{\tan \alpha}+\frac{r_{e} \cos ^{2} \alpha}{\sin \alpha}+r_{e} \sin \alpha$, the intersection point can be found between the current tool rake face and the flank face of the following tool. The feed mark high $h$ can be expressed as:

$$
h=\frac{f_{z} \tan \alpha-\frac{r_{e} \sin ^{2} \beta \tan \alpha}{\cos \beta}-r_{e} \cos \beta \tan \alpha-r_{e} \cos \alpha-r_{e} \sin ^{2} \alpha / \cos \alpha}{1-\tan \alpha \tan \beta}+r_{e}
$$


As a sinusoidal vibration is applied in the feed direction, the depth of fish scales surface structure $h_{d}$ is described by two different expressions depending on the amplitude of vibration $A_{x}$, as follows:

when $2 A_{x} \leq r_{e} \cos \beta+\frac{r_{e} \sin \beta}{\tan \alpha}+\frac{r_{e} \cos ^{2} \alpha}{\sin \alpha}+r_{e} \sin \alpha$ :

$$
h_{d}=\frac{4 r+r_{e}{ }^{2}-\left(\left(2 A_{x}-\frac{r_{e} \cos ^{2} \alpha}{\sin \alpha}-r_{e} \sin \alpha\right)^{2}-\sqrt{-4\left(2 A_{x}-\frac{r_{e} \cos ^{2} \alpha}{\sin \alpha}-r_{e} \sin \alpha\right)^{2}-2 r_{e}\left(2 A_{x}-\frac{r_{e} \cos ^{2} \alpha}{\sin \alpha}-r_{e} \sin \alpha\right)+8 r_{e}{ }^{2}}\right)}{4}
$$

when $2 A_{x}>r_{e} \cos \beta+\frac{r_{e} \sin \beta}{\tan \alpha}+\frac{r_{e} \cos ^{2} \alpha}{\sin \alpha}+r_{e} \sin \alpha$ :

$$
h_{d}=\frac{2 A_{x} \tan \alpha-\frac{r_{e} \sin ^{2} \beta \tan \alpha}{\cos \beta}-r_{e} \cos \beta \tan \alpha-r_{e} \cos \alpha-r_{e} \sin ^{2} \alpha / \cos \alpha}{1-\tan \alpha \tan \beta}+r_{e}
$$

\section{Wettability modelling}

The well-known Young's contact model describes a solid surface wetting state by considering the interaction among the three phases present, see Fig. 5. The contact angle $\theta$ can be expressed by:

$$
\cos \theta=\frac{\Upsilon_{S V}-\Upsilon_{S L}}{\Upsilon_{L V}}
$$

where $Y_{S L}, Y_{S V}$ and $Y_{L V}$ are the solid-liquid, solid-vapour and liquid-vapour interfacial tensions, respectively.

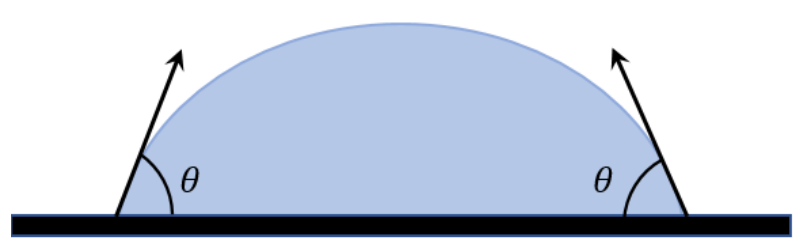

\section{Figure 5 Young's Contact Model}

However, this equation only applies to ideally smooth, uniform and rigid surfaces. The experimental value of surface contact angle is observed to differ from $\theta$. To explain this disagreement, two different theories have been put forward. Wenzel [23] developed his own liquid-surface fully contact model by considering the effect of solid surface roughness. The 
contact area between liquid and solid surface increases if one assumes that the liquid fills all the surface micro grooves (Fig. 6). As a result, solid-liquid and solid vapour energy will correspondingly change. The model predicts the Wenzel contact angle $\theta_{w}$ as:

$$
\cos \theta_{w}=r \cos \theta
$$

Where $r$ is the roughness factor (the ratio between real area and the rough surface area)

On the other hand, after investigating the surface physical and chemical properties, Cassie [24] attributed the contact angle difference to surface Gibbs free energy changing due to inhomogeneity of surface structure. He proposed a contact model where all the micro pits on the rough surface are filled with air (Fig. 7). With this assumption, the Cassie contact angle, $\theta_{c}$ is calculated as:

$$
\cos \theta_{c}=-1+f_{s}(\cos \theta+1)
$$

where $f_{s}$ is the fraction of area occupied by solids on rough surfaces.

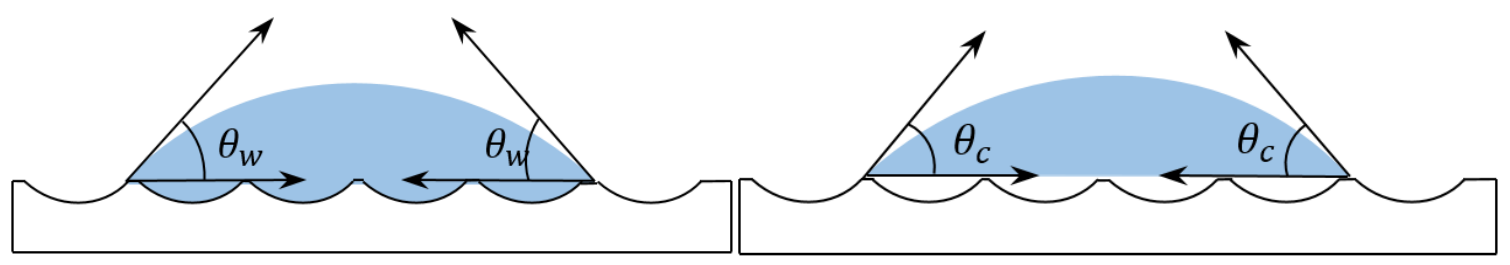

Figure 6 Wenzel Contact Model

Figure 7 Cassie Contact Model

According to these two contact models, for solid substrates of a given roughness, there exist two distinct contact angles. In fact, the wetting state can transit from Cassie model to Wenzel model for solid surfaces with pits open to the atmosphere: the droplet spreads out and fills the micro pits naturally due to its own weight and infiltration $[25,26]$. However, when the pits are closed, pockets of air can be trapped inside and cannot easily escape. After an appropriate time a state of equilibrium (stable or metastable) will be reached (Fig. 8).

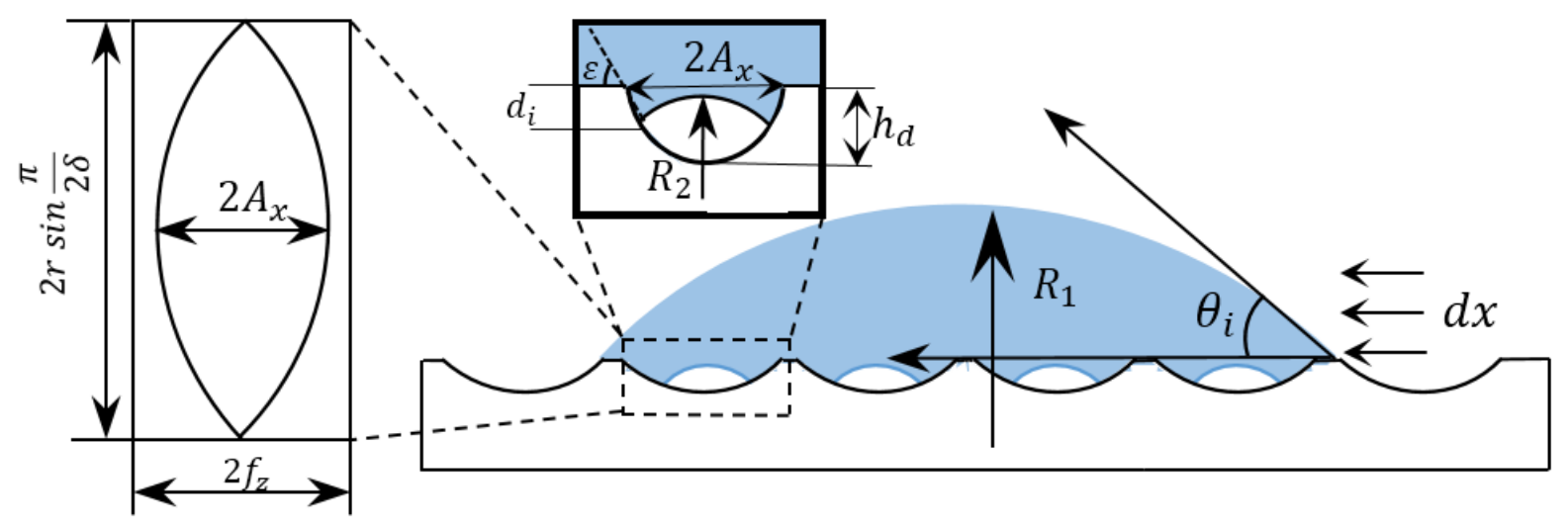


If an external disturbance causes an overall displacement $d x$ (see Fig. 8) of the water droplet, perturbing the equilibrium reached in the intermediate contact model, an increment $d G$ of the total Gibbs free energy is observed, resulting from variations in the energy of the three interfaces present:

$$
d G=d G_{S L}+d G_{S V}+d G_{L V}
$$

where $d G_{S L}, d G_{S V}$ and $d G_{L V}$ are the energy increment of the solid-liquid, solid-vapour and liquid-vapour interfaces, respectively.

By considering the fish scales surface texture in Fig. 8, Eq. (13) can be expressed as:

$$
d G=\left(1-f_{S}\right) \gamma_{L V} d x+\frac{2 d_{i} \int_{0}^{\frac{\pi r n}{60 f_{x}}} \sqrt{1+\left(\frac{60 f_{x} A_{x}}{r n} \cos \frac{60 f_{x} t}{r n}\right)^{2}} d t}{2 f_{z} r \sin \varepsilon \sin \frac{n \pi}{120 f_{x}}}\left(\Upsilon_{S L}-\Upsilon_{S V}\right) d x+\Upsilon_{L V} d x \cos \theta_{i}+f_{S}\left(\Upsilon_{S L}-\Upsilon_{S V}\right) d x
$$

where $f_{s}$ is the percentage of area occupied by solids on rough surface, given by

$$
f_{S}=1-\frac{n A_{x}}{60 f_{x} f_{z} \sin \frac{\pi}{2 \delta}}
$$

and $\varepsilon$ is the critical angle between fish scales and surface and depends on the vibration amplitude $A_{x}$ and depth of fish scales $h_{d}$.

Since the Gibbs energy is stationary at equilibrium, its derivative with respect to a perturbed parameter is zero:

$$
\lim _{d x \rightarrow 0} \frac{d G}{d x}=0
$$

By combining Eq. (14) and (16), and the expression for $f s$, a new equation for describing the contact angle in this model can be presented:

$$
\cos \theta_{i}=\left(f_{S}+\frac{d_{i} \int_{0}^{\frac{\pi r n}{60 f_{x}}} \sqrt{1+\left(\frac{60 f_{x} A_{x}}{r n} \cos \frac{60 f_{x} t}{r n}\right)^{2}} d t}{r f_{z} \sin \varepsilon \sin \frac{n \pi}{120 f_{x}}}\right) \cos \theta+f_{S}-1
$$

From Eq. (17), the static contact angle $\theta_{i}$ is determined by both vibration parameters (frequency, amplitude) and cutting parameters (spindle speed, tool geometry and feed rate).

By introducing the frequency ratio $\delta$ (defined in Eq. (3)), Eq. (17) can be simplified to: 


$$
\cos \theta_{i}=\left(1-\frac{A_{x}}{\delta f_{z} \sin \frac{\pi}{2 \delta}}+\frac{d_{i} \int_{0}^{\frac{\pi r}{\delta}} \sqrt{1+\left(\frac{\delta A_{\chi}}{r} \cos \frac{\delta t}{r}\right)^{2}} d t}{f_{z} r \sin \varepsilon \sin \frac{\pi}{2 \delta}}\right) \cos \theta-\frac{A_{x}}{\delta f_{z} \sin \frac{\pi}{2 \delta}}
$$

The value of $\sin \varepsilon$ can be calculated via trigonometry. On the other hand, the value of infiltration depth $d_{i}$ can be obtained by considering the pressure change of the air in the micropits caused by the droplet contacting the substrate. The original air pressure in the micro pits is equal to atmospheric pressure $\left(P_{0}\right)$, whereas a pressure $P_{2}$ is reached after the liquid contacts the surface and a new equilibrium is established. The pressure within the droplets $\left(P_{1}\right)$ is determined by the liquid surface tension. These quantities are related by:

$$
\begin{gathered}
P_{1}-P_{0}=\frac{2 \gamma}{R_{1}} \\
P_{2}-P_{1}=\frac{2 \gamma}{R_{2}}=\frac{2 \gamma \cos \theta}{A_{x}} \\
P_{2}=P_{0}+\frac{2 \gamma}{R_{1}}+\frac{2 \gamma \cos \theta}{A_{x}}
\end{gathered}
$$

where $\gamma$ is the liquid surface tension, $\theta$ is the contact angle, $R_{l}$ is the radius of the droplet and $R_{2}$ is the radius of the liquid in the micro pits, as shown in Fig. 8.

According to the ideal gas law, the product of air pressure and volume remains unchanged during this isothermal process. Moreover, the volume of the two states can be calculated geometrically. Consequently, the two states can be expressed as:

$$
\begin{gathered}
\frac{P_{0} \pi^{2} A_{x} h_{d} r n}{180 f_{x}}=\frac{P_{2} \pi^{2} A_{x} r n\left(h_{d}-d_{i}\right)^{3}}{135 f_{x} h_{d}{ }^{2}} \\
d_{i}=h_{d}-\sqrt[3]{\frac{3 P_{0} h_{d}^{3}}{4 P_{2}}}
\end{gathered}
$$

By substituting Eq. (23) into Eq. (18), the solid surface contact angle can be obtained.

\section{Experimental setup}

\subsection{Surface texture generation}

The experiments are conducted on a desktop precision micro milling machine (Nanowave MTS5R) which is equipped with a high speed spindle (max 80,000 rpm). The vibration stage 
is mounted on the machine as shown in Fig.9. Slots milling experiments are conducted on Al6061 alloys with vibration in the feed direction. $1 \mathrm{~mm}$ diameter uncoated carbide end mills are used, and the slot cross section dimensions are $5 \mathrm{~mm}$ wide and $50 \mu \mathrm{m}$ deep. The machining and vibration parameters are listed in Table 1.

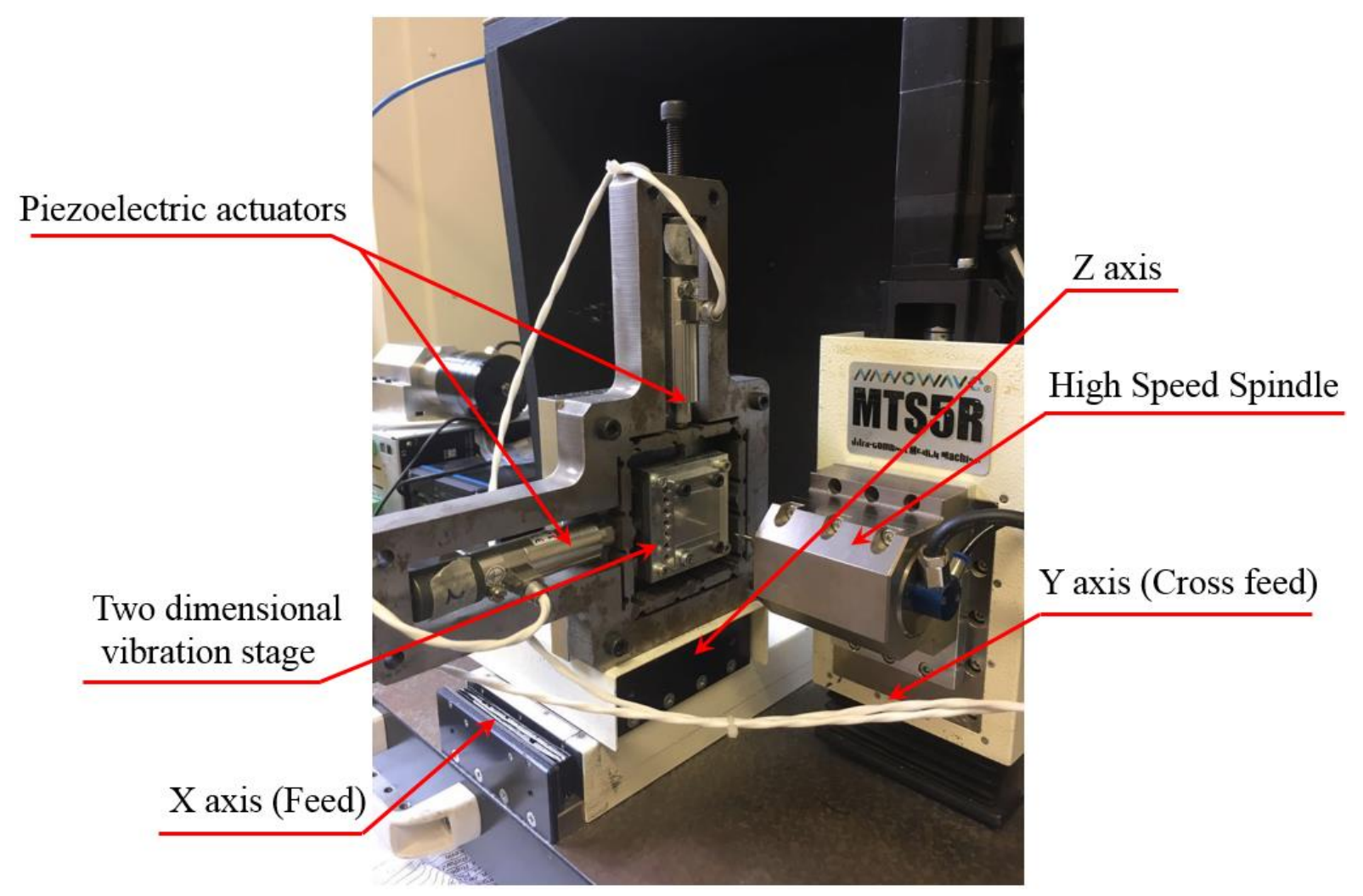

Figure 9 Layout of vibration assisted micro milling equipment

Table 1: Experiments cutting parameters

\begin{tabular}{cccccc}
\hline No & $\begin{array}{c}\text { Amplitude } \\
(\mu \mathrm{m})\end{array}$ & $\begin{array}{c}\text { Frequency } \\
(\mathrm{Hz})\end{array}$ & $\begin{array}{c}\text { Spindle speed } \\
(\mathrm{rpm})\end{array}$ & $\begin{array}{c}\text { Feed rate } \\
(\mu \mathrm{m} / \text { tooth })\end{array}$ & Frequency ratio \\
\hline 1 & 0 & 0 & 3000 & 8 & 0 \\
2 & 4 & 1950 & 3000 & 8 & 39 \\
3 & 4 & 1750 & 3000 & 8 & 35 \\
4 & 4 & 1450 & 3000 & 8 & 29 \\
5 & 4 & 1250 & 3000 & 8 & 25 \\
6 & 4 & 950 & 3000 & 8 & 19 \\
7 & 2 & 1250 & 3000 & 8 & 25 \\
8 & 6 & 1250 & 3000 & 8 & 25 \\
9 & 8 & 1250 & 3000 & 8 & 25 \\
\hline
\end{tabular}




\subsection{Surface wettability measurement}

The machined specimens are cleaned with ethanol solution in an ultrasonic cleaner. The machined surface textures are verified by an optical surface profiler and the corresponding surface contact angles are measured by using a contact angle meter (OneAttension with Theta Lite, LT100) through the sessile droplet method. The whole testing process is performed at room temperature. Three measurements were carried out at each measurement point with the amount of $5 \mu \mathrm{L} \pm 0.1 \mu \mathrm{L}$ water droplets to increase the measurement accuracy.

\section{Results and discussion}

\subsection{Machining results}

The machined surface texture is observed using white light interferometer (Zygo NewView 5200) and the testing results are shown in Fig. 10 and 11. Fig.10 shows the surface generated by the conventional micro milling without vibration assistance and clear regular circular tool marks can be observed. The results obtained from vibration assisted micro milling are shown in Fig. 11. The results obtained from different frequency ratios ranging from 19 to 39 with fixed vibration amplitude of $4 \mu \mathrm{m}$ (half of the feed per tooth) are shown in Fig. 11(a-e). It can be observed that the locus of the tool vibration are along with the cutting path, the crest (valley) of one tooth exactly overlaps with the valley (crest) of another tooth which leads to the finish scale surface textures. When the frequency ratio increases, the area of the fish scales decreases as predicted from the simulation. Fig.(b), (f), (g), (h) illustrate the surface texture with the No.2, 7, 8, 9 experiments, i.e. the vibration amplitude varying from 2 to $8 \mu \mathrm{m}$ while the frequency ratio is fixed at 25 . When the vibration amplitude increases, the relationship of one tooth crest (valley) to another's valley (crest) changes from no contact to overlap, which in turn influences the size of the fish scales unit. This agrees well with the simulation results.

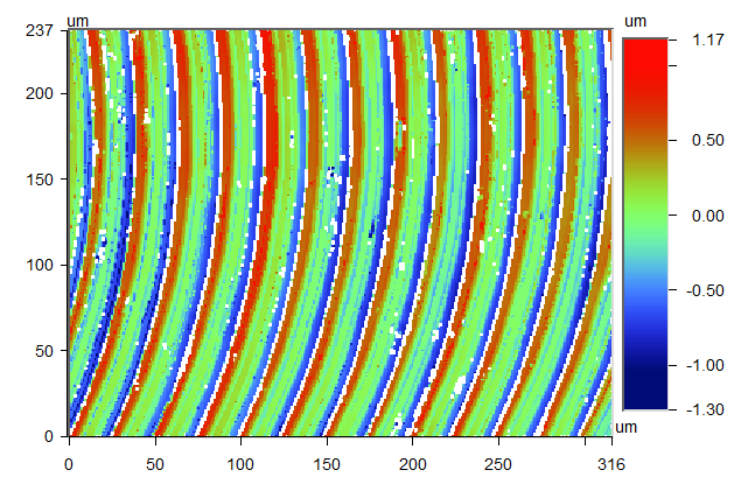


Figure 10 Surface texture of conventional micro milling

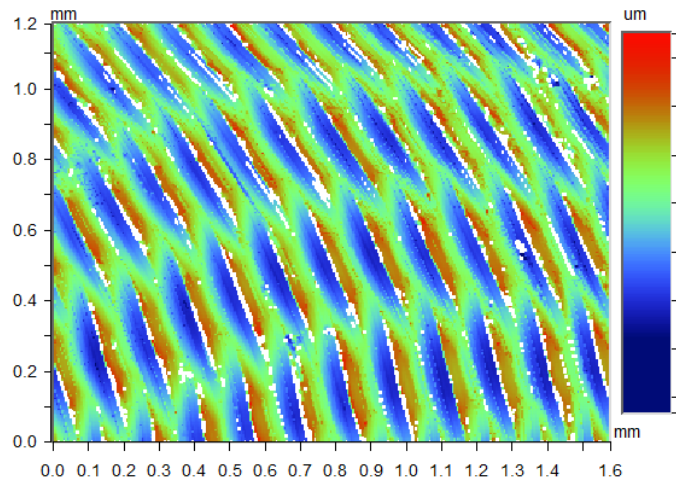

(a)

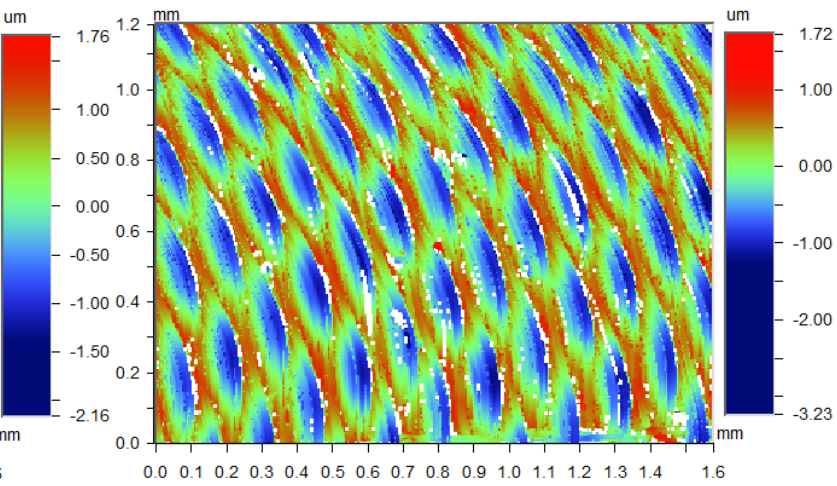

(b)

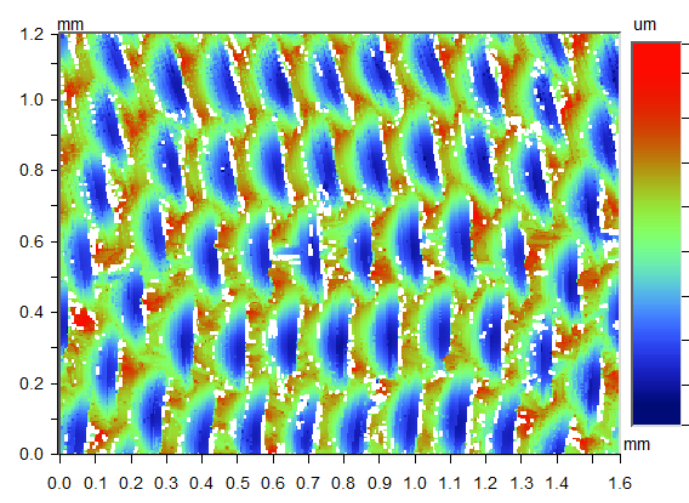

(c)

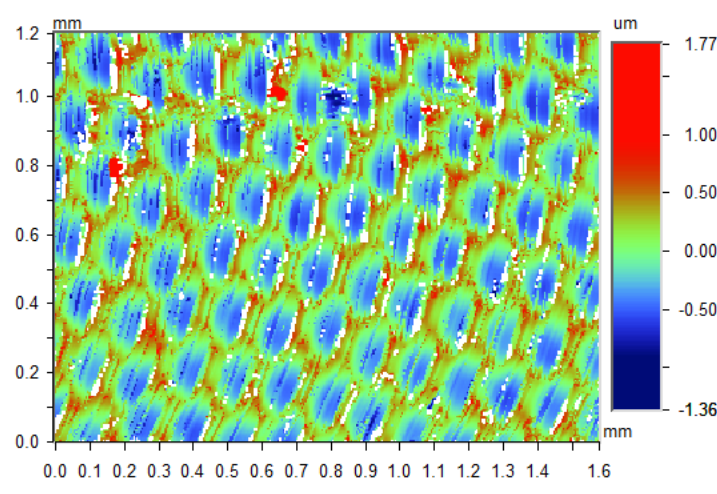

(e)

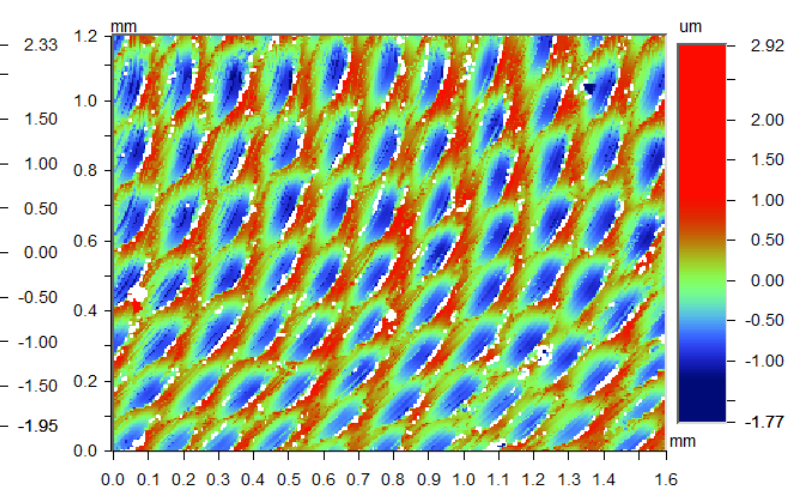

(d)

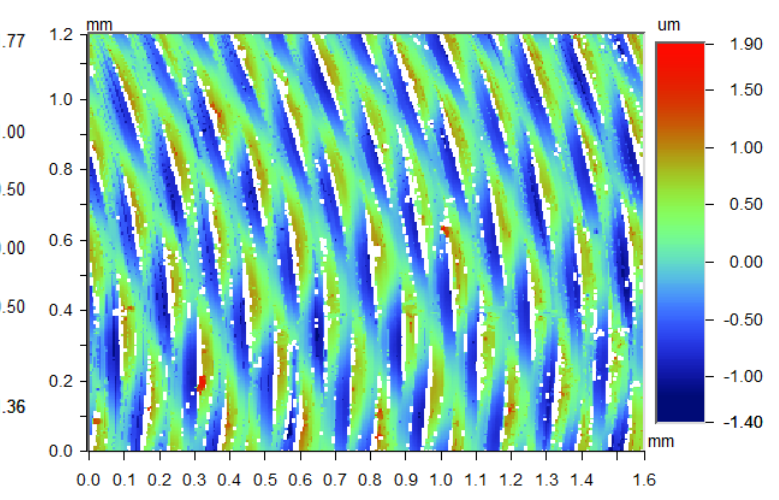

(f) 


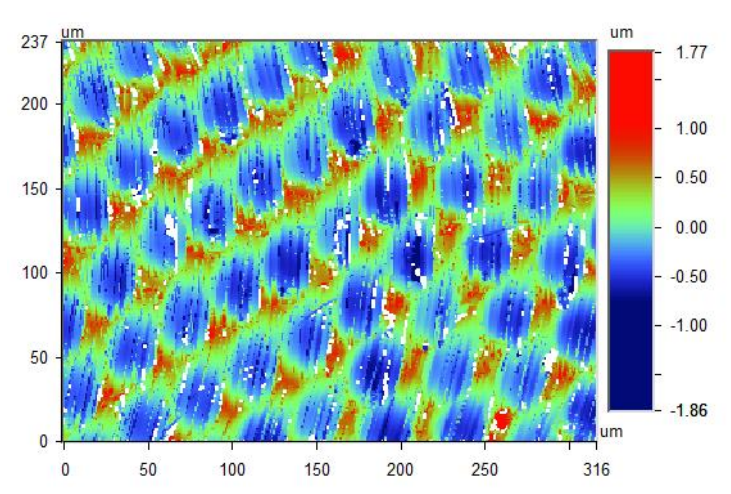

(g)

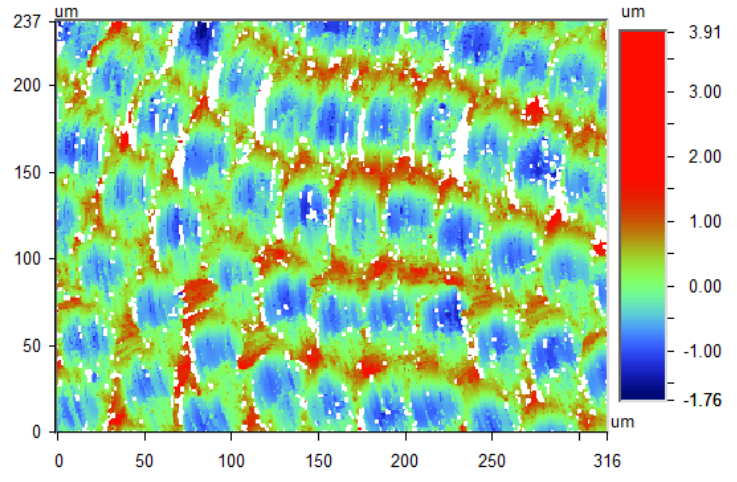

(h)

Figure 11 Fish scales surface textures with different vibration parameters: (a) Vibration amplitude 4 um with frequency ratio 19; (b) Vibration amplitude $4 \mu \mathrm{m}$ with frequency ratio 25; (c) Vibration amplitude $4 \mu \mathrm{m}$ with frequency ratio 29; (d) Vibration amplitude $4 \mu \mathrm{m}$ with frequency ratio 35; (e) Vibration amplitude $4 \mu \mathrm{m}$ with frequency ratio 39; $(f)$ Vibration amplitude $2 \mu \mathrm{m}$ with frequency ratio 25; $(\mathrm{g})$ Vibration amplitude $6 \mu \mathrm{m}$ with frequency ratio 25; $(h)$ Vibration amplitude $8 \mu \mathrm{m}$ with frequency ratio 25.

\subsection{Wettability testing results}

As discussed in the previous section, the surface wettability can be quantitatively measured and compared through contact angles. Fig. 12 illustrates contact angle results with different vibration parameters. It can be observed that the contact angle in the conventionally machined surface was measured as $89^{\circ}$, whereas all the surfaces machined by vibration assisted micro milling have lower contact angle values ranging from $33^{\circ}$ to $57^{\circ}$, which indicates that a more hydrophilic surface has been produced by vibration assisted milling.

The contact angle obtained from machining experiments was compared with simulation results obtained from the proposed contact model, as well as both Wenzel's and Cassie's contact models(see Fig. 13). Compared with the results of Wenzel's and Cassie's contact models, the proposed contact model provides more accurate contact angle prediction in both frequency ratio domain and vibration amplitude domain. In Fig. 13 (a), as frequency ratio increases from 19 to 39, the variation of corresponding experimental contact angle shows a linear increase from $33^{\circ}$ to $57^{\circ}$, which follows almost the same trend as the theoretical prediction. As shown in the tool motion simulation results (see Fig.3), the area of the micro unit reduces when the frequency ratio increases, which changes the percentage of area occupied by solids on rough surface and the filtration area in the fish scales structure. According to Eqs. (13-23), the value of contact angle increases and the surface wettability turns to be more hydrophobic. In addition, it is reported that the contact angle is one of the important factors 
indicating the surface free energy, where smaller surface free energy corresponds to higher contact angles [8]. This implies that increasing the frequency ratio will decrease the surface free energy. Fig. 13 (b) shows the effect of vibration amplitude for both testing and theoretical data when the frequency ratio is 25 . Generally, the contact angle decreases with the vibration amplitude and reaches the lowest value (around $33^{\circ}$ ) at $8 \mu \mathrm{m}$ and the highest value (around $45^{\circ}$ ) at $2 \mu \mathrm{m}$, which agrees well with the theoretical data. This is due to higher vibration amplitude causing a larger size of fish scales unit, which affects the infiltration area and the air pressure in the micro structure. As a result, a lower contact angle and an increased surface free energy can be obtained.
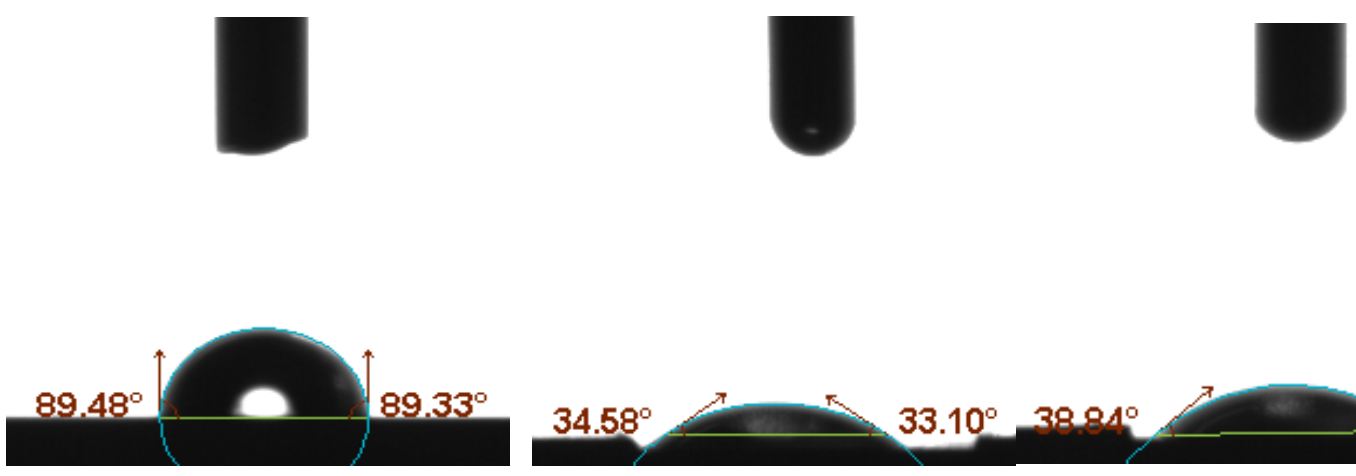

(a)
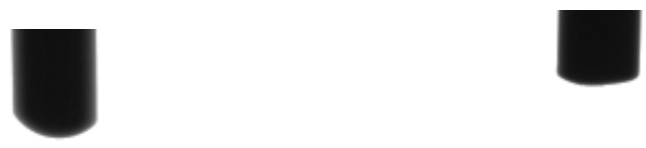

(b)

(c)
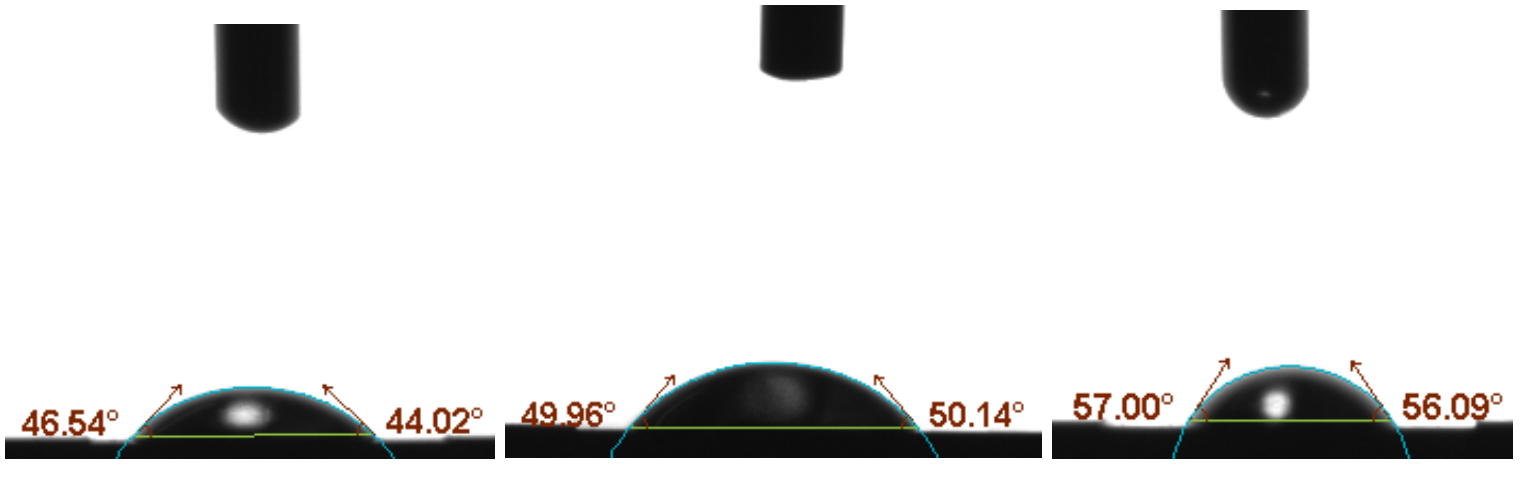

(d)

(e)

(f) 
Figure 12 Contact angle measurement results: (a) conventional micro milling (b) Vibration amplitude $4 \mu \mathrm{m}$ with frequency ratio 19; (c) Vibration amplitude $4 \mu \mathrm{m}$ with frequency ratio 25; (d) Vibration amplitude $4 \mu \mathrm{m}$ with frequency ratio 29; (e) Vibration amplitude $4 \mu \mathrm{m}$ with frequency ratio 35; $(f)$ Vibration amplitude $4 \mu \mathrm{m}$ with frequency ratio 39; $(\mathrm{g})$ Vibration amplitude $2 \mu \mathrm{m}$ with frequency ratio 25; (h) Vibration amplitude $6 \mu \mathrm{m}$ with frequency ratio 25; (i) Vibration amplitude $8 \mu \mathrm{m}$ with frequency ratio 25 ;

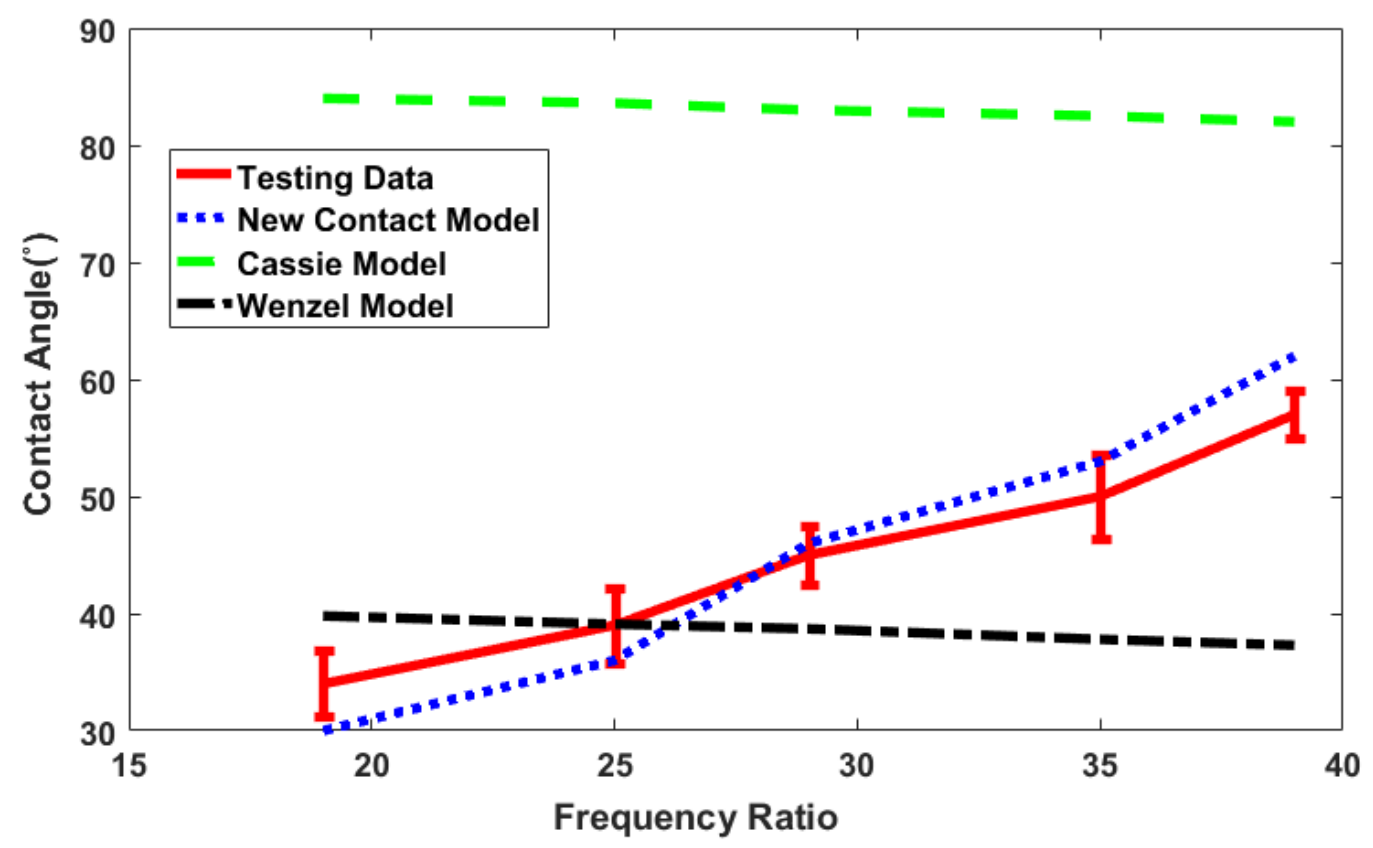

(a) 


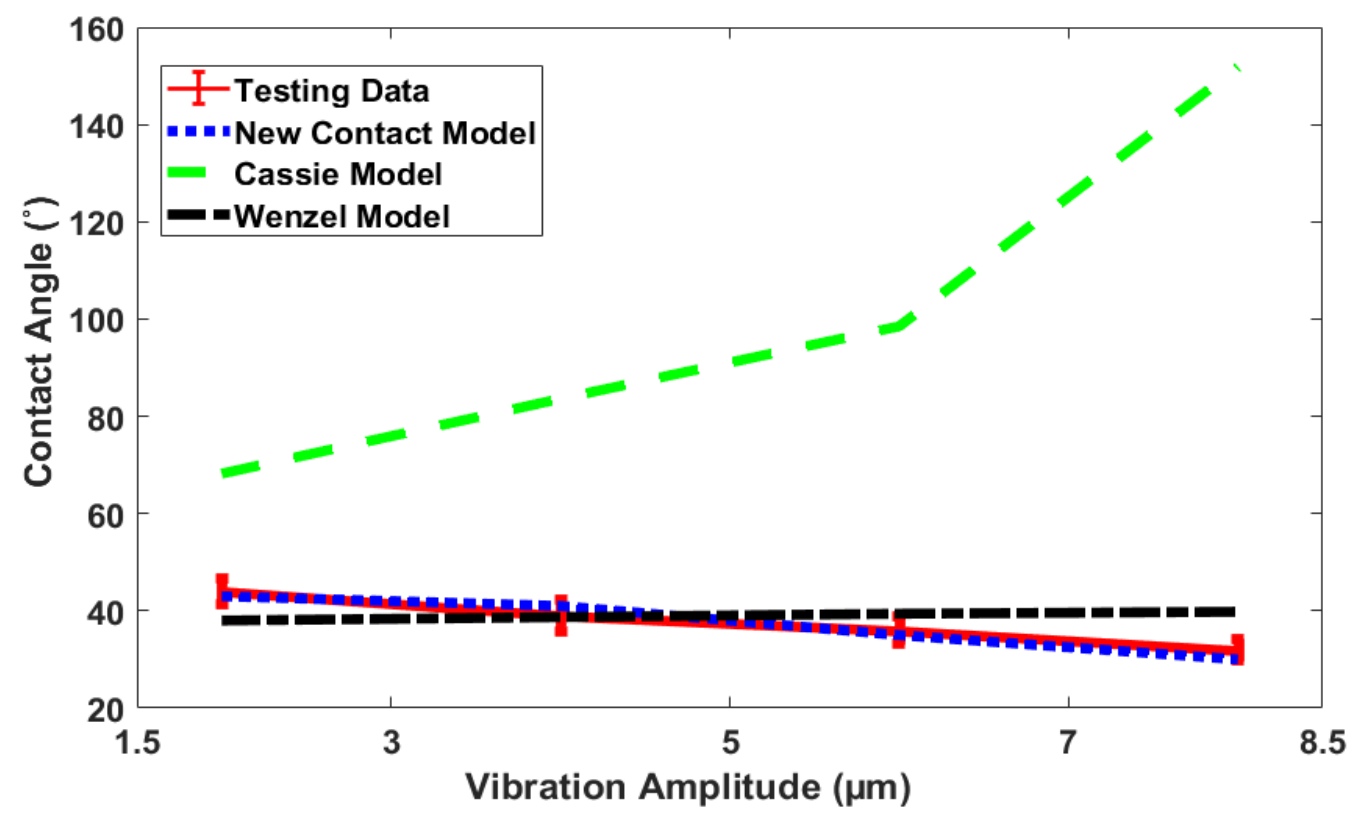

(b)

Figure 13 Contact angle results between experimental and theoretical data, (a) data of frequency ratio domain with the vibration amplitude fixed at $4 \mu \mathrm{m}$; (b) data of vibration amplitude domain with frequency ratio fixed at 25.

\section{Conclusions}

In this paper, vibration assisted micro milling is used to produce surface textures with controllable wettability. The influence of the frequency ratio (vibration frequency over spindle speed) and the vibration amplitude on the surface texture and its relationship with hydrophilicity are investigated. The following conclusions can be drawn:

1) Surfaces with fish scales textures can be produced by using appropriate machining and vibration parameters. The relationship between the machining and vibration parameters are established in the surface generation model. The size of the fish scales unit can be controlled by the frequency ratio and the vibration amplitude.

2) A contact model is developed to predict the hydrophilicity of fish scales textured surfaces by considering the effects of trapped air pressure in the surface microstructure, infiltration area and the percentage of area occupied by solids on a rough surface.

3) The wettability testing results on the machined surface show that the proposed contact model can predict the contact angle accurately.

4) The results also indicate that the fish scales textured surface turns to more hydrophilic and a surface with controllable wettability can be obtained by optimizing the machining and vibration parameters. 


\section{Acknowledgment}

The authors gratefully acknowledge financial support of the Engineering and Physical Sciences Research Council (EP/M020657/1).

\section{References}

[1] T. Firincioglu, M.J. Blunt, D. Zhou, Three-phase flow and wetability effects in triangular capillaries, Colloids Surfaces A Physicochem. Eng. Asp. 155 (1999) 259-276. doi:10.1016/S09277757(99)00039-4.

[2] T. Young, An Essay on the Cohesion of Fluids, Philos. Trans. R. Soc. London. 95 (1805) 6587. doi:10.1098/rstl.1805.0005.

[3] X. Zhou, N.R. Morrow, S. Ma, Interrelationship of Wettability, Initial Water Saturation, Aging Time, and Oil Recovery by Spontaneous Imbibition and Waterflooding, SPE J. 5 (2000) 199-207. doi:10.2118/62507-PA.

[4] R. Li, J. Chen, Studies of wettability of medical PVC by remote nitrogen plasma, Plasma Sci. Technol. 8 (2006) 325-328. doi:10.1088/1009-0630/8/3/17.

[5] J.. Buckley, K. Takamura, N.. Morrow, Influence of Electrical Surface Charges on the Wetting Properties of Crude Oils, Spe 16964. (1989) 332-340. doi:10.2118/16964-PA.

[6] A. Borruto, G. Crivellone, F. Marani, Influence of surface wettability on friction and wear tests, Wear. 222 (1998) 57-65. doi:10.1016/S0043-1648(98)00256-7.

[7] W. Li, A. Amirfazli, A thermodynamic approach for determining the contact angle hysteresis for superhydrophobic surfaces, J. Colloid Interface Sci. 292 (2005) 195-201. doi:10.1016/j.jcis.2005.05.062.

[8] S. Zhenyu, L. Zhanqiang, S. Hao, Z. Xianzhi, Prediction of contact angle for hydrophobic surface fabricated with micro-machining based on minimum Gibbs free energy, Appl. Surf. Sci. 364 (2016) 597-603. doi:10.1016/j.apsusc.2015.12.199.

[9] H.-C. Yang, Y.-F. Chen, C. Ye, Y.-N. Jin, H. Li, Z.-K. Xu, Polymer membrane with a mineral coating for enhanced curling resistance and surface wettability, Chem. Commun. 51 (2015) 1277912782. doi:10.1039/C5CC03216D.

[10] F. Topuz, M. Möller, J. Groll, Covalently layer-by-layer assembled homogeneous nanolayers with switchable wettability, Polym. Chem. 6 (2015) 4690-4697. doi:10.1039/C5PY00515A.

[11] J.B. Lee, H.R. Gwon, S.H. Lee, M. Cho, Wetting Transition Characteristics on Microstructured Hydrophobic Surfaces, Mater. Trans. 51 (2010) 1709-1711. doi:10.2320/matertrans.M2010118.

[12] M. V. Rukosuyev, J. Lee, S.J. Cho, G. Lim, M.B.G. Jun, One-step fabrication of superhydrophobic hierarchical structures by femtosecond laser ablation, Appl. Surf. Sci. 313 (2014) 411-417. doi:10.1016/j.apsusc.2014.05.224. 
[13] S. Razi, M. Mollabashi, K. Madanipour, Laser processing of metallic biomaterials: An approach for surface patterning and wettability control, Eur. Phys. J. Plus. 130 (2015) 1-12. doi:10.1140/epjp/i2015-15247-5.

[14] A.D. Sommers, A.M. Jacobi, Creating micro-scale surface topology to achieve anisotropic wettability on an aluminum surface, J. Micromechanics Microengineering. 16 (2006) 1571-1578. doi:10.1088/0960-1317/16/8/018.

[15] a R. Burgers, J.H. Bultman, C. Beneking, W. a Nositschka, O. Voigt, H. Kurz, Silicon solar cells textured by reactive ion etching with natural lithography, 16th Eur. Photovolt. Sol. Energy Conf. Glas. (2000) VA1/57.

[16] W. Chen, L. Zheng, D. Huo, Surface texture formation by non-resonant vibration assisted micro milling, J. Micromechanics Microengineering. 28 (2018) 025006.

[17] J. Janghorbanian, M.R. Razfar, M.M.A. Zarchi, Effect of cutting speed on tool life in ultrasonic-assisted milling process, Proc. Inst. Mech. Eng. Part B J. Eng. Manuf. 227 (2013) 1157-1164. doi:10.1177/0954405413483722.

[18] D.E. Brehl, T.A. Dow, Review of vibration-assisted machining, Precis. Eng. 32 (2008) 153172. doi:10.1016/j.precisioneng.2007.08.003.

[19] H. Lian, Z. Guo, Z. Huang, Y. Tang, J. Song, Experimental research of Al6061 on ultrasonic vibration assisted micro-milling, in: Procedia CIRP, 2013: pp. 561-564. doi:10.1016/j.procir.2013.03.056.

[20] X.H. Shen, J.H. Zhang, H. Li, J.J. Wang, X.C. Wang, Ultrasonic vibration-assisted milling of aluminum alloy, Int. J. Adv. Manuf. Technol. 63 (2012) 41-49. doi:10.1007/s00170-011-3882-5.

[21] G.L. Chern, Y.C. Chang, Using two-dimensional vibration cutting for micro-milling, Int. J. Mach. Tools Manuf. 46 (2006) 659-666. doi:10.1016/j.ijmachtools.2005.07.006.

[22] W.X. Xu, L.C. Zhang, Ultrasonic vibration-assisted machining: principle, design and application, Adv. Manuf. 3 (2015) 173-192. doi:10.1007/s40436-015-0115-4.

[23] R.N. Wenzel, Resistance of solid surfaces to wetting by water, Ind. Eng. Chem. 28 (1936) 988-994. doi:10.1021/ie50320a024.

[24] A.B.D. Cassie, S. Baxter, Wettability of porous surfaces, Trans. Faraday Soc. 40 (1944) 546. doi:10.1039/tf9444000546.

[25] C.I. Park, H.E. Jeong, S.H. Lee, H.S. Cho, K.Y. Suh, Wetting transition and optimal design for microstructured surfaces with hydrophobic and hydrophilic materials, J. Colloid Interface Sci. 336 (2009) 298-303. doi:10.1016/j.jcis.2009.04.022.

[26] X. Feng, L. Jiang, Design and creation of superwetting/antiwetting surfaces, Adv. Mater. 18 (2006) 3063-3078. doi:10.1002/adma.200501961. 